\title{
How to recognize the traces left on a crime scene by a 3D-printed Liberator?
}

\section{Part 1. Discharge, exterior ballistic and wounding potential}

\author{
Honsberger H. ${ }^{1}$, Rhumorbarbe D. ${ }^{1}$, Werner D. ${ }^{1}$, Riva F. ${ }^{2}$, Glardon M. ${ }^{2}$, \\ Gallusser A. ${ }^{1}$, Delémont $\mathrm{O}^{1}$. \\ ${ }^{I}$ Ecole des sciences criminelles, University of Lausanne, Lausanne, Switzerland. \\ ${ }^{2}$ Institut für Rechtsmedizin, University of Bern, Bern, Switzerland
}

\begin{abstract}
The Liberator is a firearm that can be manufactured from its blueprints, using a 3D-printer. This weapon made of nineteen pieces - eighteen in printed plastic and one metallic nail - raises questions such as its ability to fire a round, its wounding potential and the traces produced by its discharge. In particular, knowledge must be gained to infer that a 3D-printed handgun was used, reconstruct the shooting event involving such handgun, and gather information related to the type of 3D-printed handgun used. This study focused on the traces that could orientate forensic investigations when the use of a 3D-printed Liberator is suspected. In a first step, the Liberator was investigated to study its behaviour during the discharge and characterize traces produced by the discharge. To fulfil this goal, some Liberators were printed and assembled. Six Liberators fired a round. The discharge of the weapons was done under specific conditions allowing to collect ballistics data and traces produced by the shooting.

The results showed that the barrel tended to break between the ignition of the primer and the moment the projectile exited the muzzle. The speed of the projectiles reached $140 \mathrm{~m} / \mathrm{s}$ when the barrel broke, while it was about $170 \mathrm{~m} / \mathrm{s}$ when barrel remained intact. The trajectory of the projectiles was sometimes disrupted, and the projectile tumbled on itself. It was thus very difficult to characterize the trajectory. The cavity wound caused by the fastest bullet was typical of a handgun wound firing a FMJ projectile (penetration of $21 \mathrm{~cm}$ in ballistics soap). On the other hand, the cavity caused by the slowest bullet was more representative of a splinter wound (penetration of $14 \mathrm{~cm}$ in ballistics soap). The study of gunshot residues collected on adhesive targets showed the presence of unburnt particles and small perforations caused by polymer pieces that concentrated around the entry holes.
\end{abstract}

Keywords: Additive manufacturing ; Homemade firearm ; Handgun

\section{Introduction}

In May 2013, the blueprints of a firearm were made available by Cody Wilson, founder of Defense Distributed, a collaborative community aiming at sharing these files. What makes the Liberator very different from classical firearms is that it is made of polymer and produced by a 3D-printer. The manufacture of such handgun comes within reach of every person who has access to such technology. As the Liberator became very popular in the first few days, American authorities asked Wilson to remove the plans of the Liberator from the Internet, among other blueprints he also designed. However, it is believed that the files had been downloaded about 100,000 times during the first two days they were available. Today, it is still possible to find the blueprints, on online torrent file indexes or on cryptomarkets hosted on the Dark web [1]-[4]. Such handguns have been encountered during investigations on scenes or searches by police forces [1].

The Liberator was named after the FP-45 Liberator, chambered for .45 ACP cartridges, which was manufactured during the Second World War to be distributed to the resistance forces into the Axis-occupied territories [5]. However, both the aspect and the inner parts of the FP-45 are different from the printed version. The printed Liberator has the aspect of a regular handgun. Its dimensions are $216 \mathrm{~mm}$ by $16 \mathrm{~mm}$, the length of the barrel being $64 \mathrm{~mm}$. It is made of eighteen printed polymer pieces and one metal nail. Since the nail is used as firing pin, it is the only non-polymer piece to ensure the proper percussion of the primer. 
When releasing the blueprints, Cody Wilson also uploaded a video of himself successfully firing a round with the Liberator. Consequently, several law enforcement agencies tested the Liberator, concluding that the weapon may be functional depending on the quality of the plastic used to print it [2]. Moreover, experiments made within the $\mathrm{ATF}^{1}$ showed that a bullet fired by a Liberator could be deadly, as it was able to penetrate soft tissues for several inches or a human skull [6]. These tests illustrate the functionality of the printed Liberator, causing police forces to consider it as a possible weapon allowing to fire at least one round [2].

Nowadays, 3D-printing technologies are widely available, as the cheapest 3D-printers cost less than a thousand US-dollars [1]. From a legal perspective, regulating firearms 3D-printing is a challenge. So far, controlling the printing of pieces that will form a handgun when assembled is almost impossible. Besides, since the functional blueprints are now easily accessible online, they cannot be regulated either. However, despite the difficulty to control the production of 3D-printed firearms, national laws dealing with production of weapons already apply to these weapons as well [2].

Given their composition and their mechanism, it is likely that 3D-printed firearms will produce specific traces when discharged. In the context of a forensic investigation, understanding the type of traces that can be created, transferred and found, as well as their location and origin, is a crucial step for reconstruction purposes. As for usual firearms, the weapon itself might bear traces of the action, as well as the environment or the target.

Only a few studies have been published about the discharge of printed firearms. Crowe's research showed that the gunshot residues induced by the discharge of a Liberator encompass small particles of polymer. According to their size and shape, they are likely to be fragments torn out of the Liberator. Polymer smears were also found on the sides of bullets and cartridge cases. This information provides investigative leads when no firearm is found on a crime scene [7]. The study of Black and al. - who printed firearms and fired ammunitions with the assembled pieces - illustrates the detection of polymer from the barrels on the bullets and cartridge cases, using direct analysis in real time coupled with mass spectrometry (DART-MS) [8].

Our study aims at developing a test-firing process to analyse the discharge of polymer firearms. The impact of the discharge on the weapon itself, the speed and behaviour of the bullets as well as the effect on the target will be described in this first article. A second one will address the manifold dimension of the traces produced by the discharge of such a weapon.

\section{Materials and method}

\subsection{Printing and assembling process}

\subsubsection{Plans and printing process}

The bundle "Liberator" is composed of a Defense Distributed license, 16 STL files, ${ }^{2}$ a "ReadMe.txt"- in both English and Chinese - with instructions regarding the assembling, and a scheme of a handgun. These elements suggest that the blueprints are a copy of the files originally shared by Defense Distributed in 2013.

Two versions of the barrel - threaded and unthreaded - are available, depending on the kind of printer used. The former was selected for this study, in agreement with the specifications of the printer. The names of the files contain the number of times they should be printed to build the Liberator (i.e. "Spring ( $x 2$ ).stl" has to be printed twice).

An authorization to print the weapons for this study was issued by the local competent authorities, strengthening the legal basis for conducting this research activity.

\footnotetext{
${ }^{1}$ Bureau of Alcohol, Tobacco, Firearms and Explosives, in the United States of America.

2 STL (for stereolithography) is a file format commonly used in computer-aided design applications such as rapid prototyping or 3D-printing. STL files include the geometry of the outer surface of three-dimensional objects [9].
} 
The printer used to manufacture the Liberator is a Stratasys Fortus $400 \mathrm{mc}$ working with the software Insight, both distributed by Stratasys, Ltd. It is a fused deposition modelling printer, meaning that it relies on the deposition (layer by layer) of melted polymer in a chamber following the chosen numeric plans, until the piece is completed [1] [3]. The specificities of the Stratasys Fortus $400 \mathrm{mc}$ includes a layer thickness of $0.13 \mathrm{~mm}$ and a tempered room, which allows a better cohesion between the different layers ${ }^{3}$.

The polymer used is ABS M30, a modified ABS (acrylonitrile butadiene styrene) - sold by Stratasys, Ltd. - and considered as 25 to 70 percent more resistant than standard ABS used for 3D-printing ${ }^{4}$. Assembling a Liberator requires the printing of several pieces, as illustrated in Figure 1.

Nine complete Liberators were printed following the original instructions file. Six of them were designed to fire .380 Auto ammunition. In order to test a smaller calibre, three more barrels - suitable for 25 Auto cartridges were also designed by the staff in charge of printing the different pieces. This new version was obtained by modifying the original plans. The interior diameter of the original barrel was reduced, while the outer measurements remained unchanged.

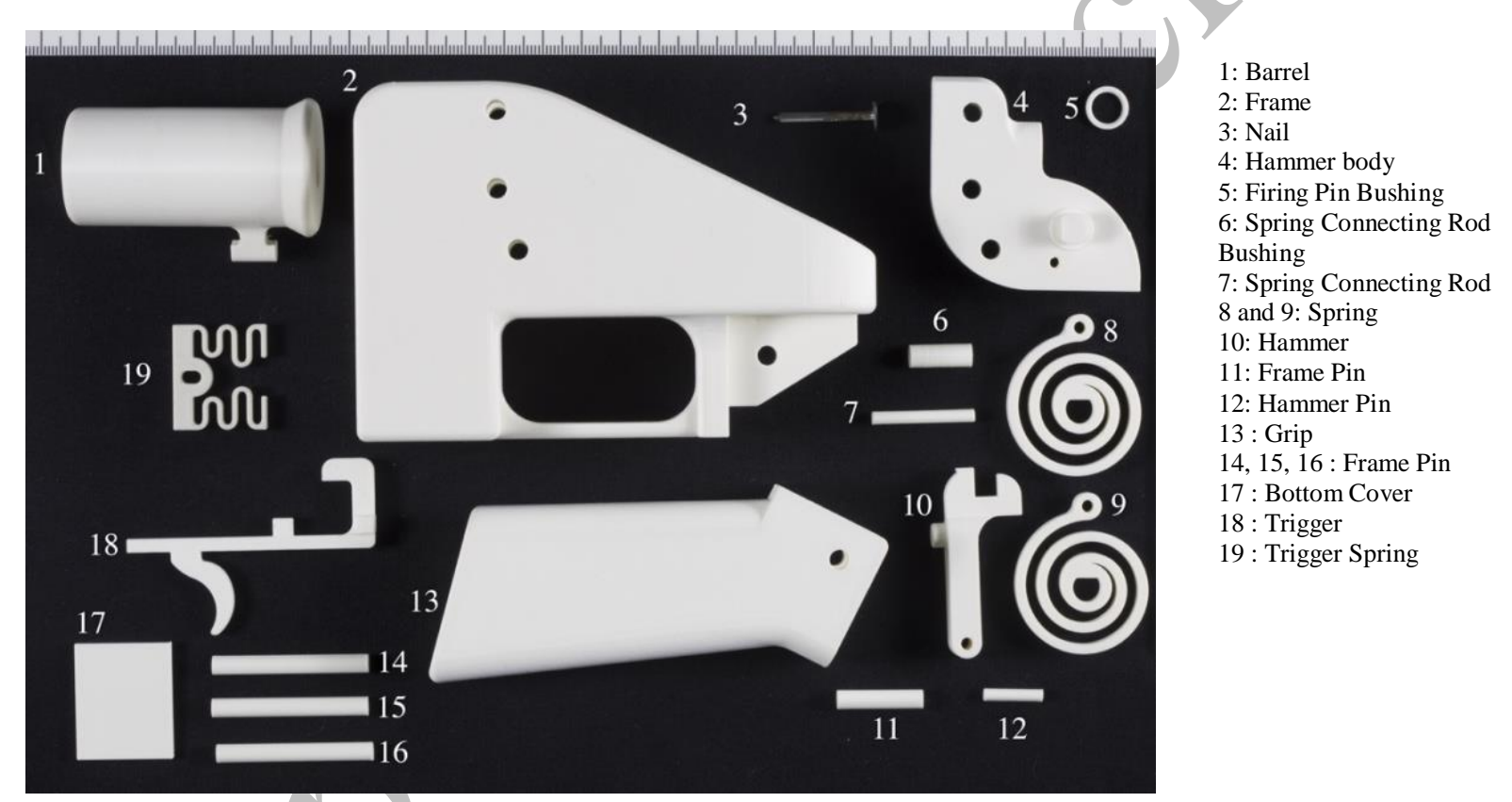

\subsubsection{Assembling process}

The "ReadMe.txt" file included in the bundle gives step-by-step instructions to assemble a Liberator. Briefly, the process consists of building the different groups and assembling them together. The hammer group (pieces $\mathrm{N}^{\circ} 4$ 10 and 12) is built first. The trigger group (pieces $\mathrm{N}^{\circ} 18,19$ ) and the hammer group are then inserted in the frame and fixed with the pins, as well as the grip. Since the ammunition must be manually inserted into the barrel, this

\footnotetext{
${ }^{3}$ Fortus 360mc-400mc User Guide, version 107166-0014_REV_A January 2016

${ }^{4}$ Information file MSS_FDM_ABSM30_0517a, issued by Stratasys, Ltd.
} 
piece is adjusted before the discharge by locking it on the frame with a rotation. The fully assembled Liberator is illustrated in Figure 2.

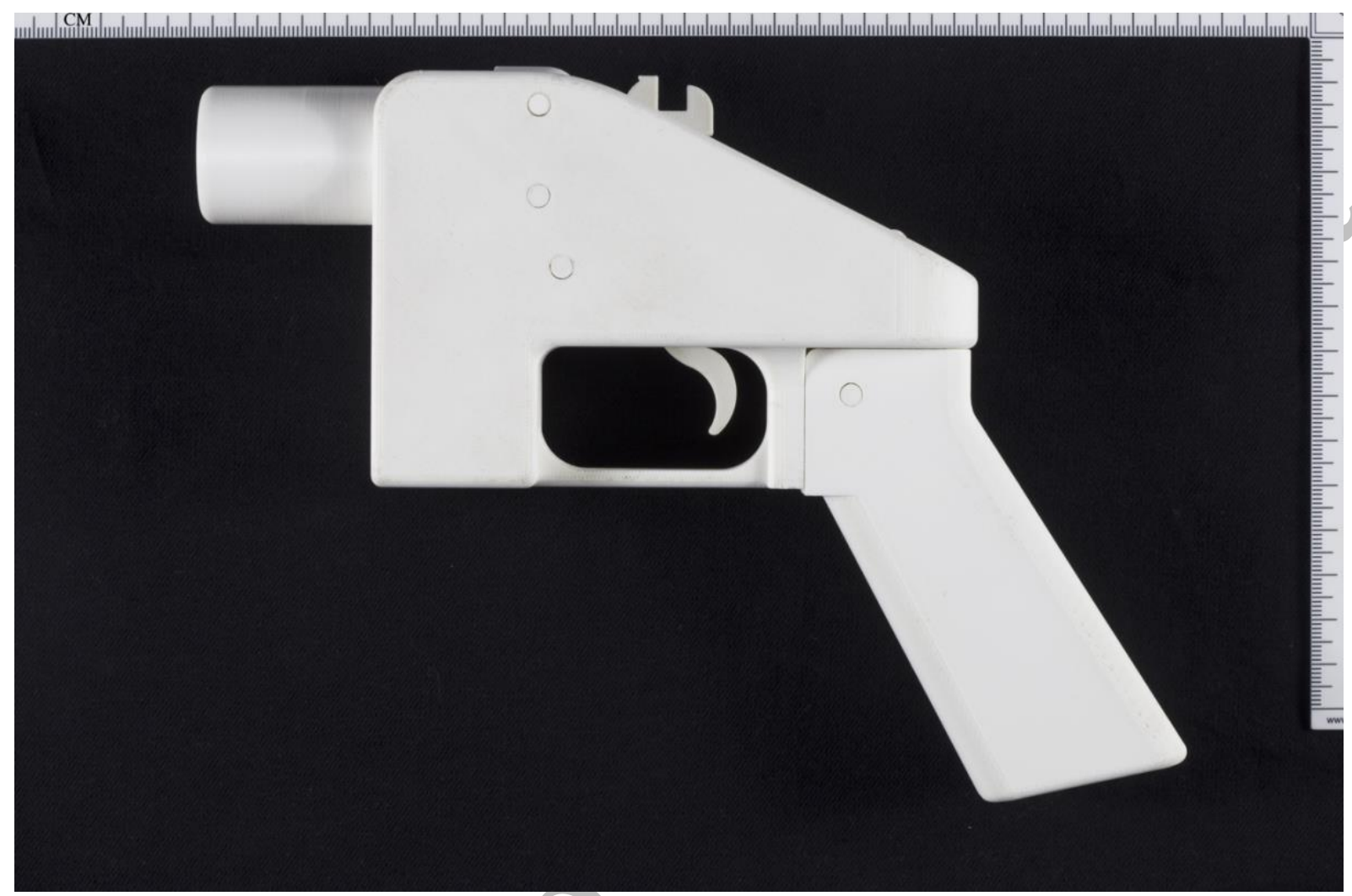

Figure 2: An assembled Liberator.

During our experiments, we noticed that springs tended to break during the assembling process due to the pressure that needs to be manually applied on them. Consequently, only six Liberator were assembled to conduct the experiments: five with a .380 Auto and one with the .25 Auto barrel.

\subsection{Test-firing process}

\subsubsection{Procedure}

Two types of Magtech ammunition were used for the experiments: .380 Auto cartridges model 380A (FMJ, 95 grains) and .25 Auto cartridges model 25A (FMJ, 50 grains). For comparison purposes, shots were also fired with conventional semi-automatic pistols: a .380 Auto Beretta 86 (barrel length: $111 \mathrm{~mm}$ ) and a .25 Auto CZ 45 (barrel length: $63 \mathrm{~mm}$ ).

The same boxes of ammunitions were used for all discharges, including the comparison firings with the Beretta 86 and the CZ 45.

All discharges were undertaken in an indoor shooting range cleared from any objects that could produce contamination. All elements being consecutive to the discharge of a 3D-printed firearm were individually collected.

The full setup of the shooting process is described in Figure 3. Regarding the impact on the target, gunshot residues (GSR) were collected using a plastic adhesive sheet. The muzzle of the Liberator and the adhesive sheet 
were $80 \mathrm{~cm}$ apart. Note that the adhesive sheet was not included for every discharge since its presence might disturb other instruments (see below).

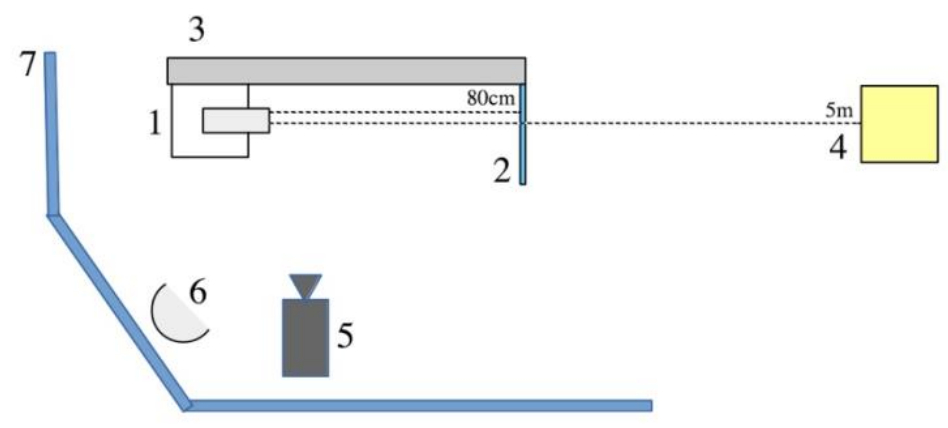

\author{
1: Liberator on the rest \\ 2: Adhesive tape to collect the gunshot residues \\ 3: Mirrors at $45^{\circ}$ \\ 4: Target (ballistics soap or Kevlar) \\ 5: High-speed camera \\ 6: Doppler Radar \\ 7: Protection glass
}

Figure 3: Test firing setup.

The Liberator was set on the ransom rest and the ammunition was inserted into the barrel. The barrel itself was locked onto the frame. The hammer was then pulled backwards and locked by the trigger mechanism. For safety reasons, the Liberator was discharged by pulling a string attached to the trigger. Pulling the trigger released the hammer from the trigger mechanism allowing it to strike the firing pin which, in turn, stroke the primer cap.

\title{
2.2.2. Measurements and recordings
}

Table 1 shows the successive experiments, as well as the measurements carried out for each of them.

\begin{tabular}{|c|c|c|c|}
\hline Number & Ammunition & $\begin{array}{c}\text { Adhesive sheet for GSR } \\
\text { collection }\end{array}$ & Target \\
\hline 1 & .380 Auto & No & Kevlar® sheets \\
2 & .380 Auto & Yes & Ballistics soap \\
3 & .380 Auto & No & Kevlar® sheets \\
4 & .380 Auto & Yes & Ballistics soap \\
5 & .380 Auto & Yes & Ballistics soap \\
6 & .25 Auto & Yes & Ballistics soap \\
\hline
\end{tabular}

Table 1: shooting process.

For each experiment, the exit velocity at the muzzle was measured using a Doppler radar (Infinition BR3503). The Doppler radar position with regard to the trajectory (see Figure 3) has been taken into account for the velocity measurements.

A high-speed camera (Fastcam Photron SAX-2) was placed on the side of the ransom rest. Additionally, a mirror with a 45-degree angle was placed on the other side of the ransom rest. This set-up allowed the recording of the shooting process as well as the observation of the subsequent trajectory of the projectile, from the side and from the top. The discharge was recorded with an image acquisition frequency of 13'500 frames per second (fps).

Subsequent observations of the adhesive sheets were undertaken using several magnifications with a Leica M125 (Leica Microsystems, Germany) and a Docucenter Nirvis (Projectina, Switzerland) working with the software PIA 7000 (with wavelengths ranging from $254 \mathrm{~nm}$ to $715 \mathrm{~nm}$ ). Glycerine ballistics soap (Mettler-Seifen AG, Switzerland) with a density of $1080 \mathrm{~kg} / \mathrm{m}^{3}$ were used to recover the bullets. The ballistics soap and the Liberator were $5 \mathrm{~m}$ apart. In contrast to ballistic gelatine, glycerine soap provides an easier and permanent visualisation of the cavity caused by the projectile [10]. Then, the ballistics soap was cut in the middle of the cavity to measure the depth of the penetration and document the cavity. Two projectiles were fired in ballistics soap (shots $\mathrm{N}^{\circ} 2$ and 
4), and four were stopped with Kevlar ${ }^{\circledR}$ sheets ( $\left.\operatorname{shots~} \mathrm{N}^{\circ} 1,3,5,6\right)$ so that the condition of the bullet could be studied more accurately.

\section{Results}

Table 2 gives an overview of the results obtained for the 6 discharges. More details are provided in the following sections.

\begin{tabular}{|c|c|c|c|c|}
\hline Liberator number & Ammunition & $\begin{array}{c}\text { Exit velocity at the } \\
\text { muzzle [m/s] }\end{array}$ & $\begin{array}{c}\text { Condition of the } \\
\text { barrel after the } \\
\text { discharge }\end{array}$ & $\begin{array}{c}\text { Penetration in the } \\
\text { ballistics soap } \\
\text { [cm] }\end{array}$ \\
\hline 1 & .380 Auto & 167 & Intact & \\
2 & .380 Auto & 138 & Broken & 14 \\
3 & .380 Auto & 138 & Broken & - \\
4 & .380 Auto & 172 & Intact & 21 \\
5 & .380 Auto & - & Broken & - \\
6 & .25 Auto & - & Broken & - \\
\hline
\end{tabular}

Table 2: exit velocity, state of the barrel after the discharge and depth of penetration in the ballistics soap.

\subsection{Impact of the discharge on the weapon}

Among the six discharges, the barrel was found intact in two cases (shots 1 and 4). In four cases, the barrel broke and the frame was cracked (shots 2, 3, 5, 6). The condition of the Liberator after the discharge was variable, as illustrated in Figure 4. On the Liberator 1, only one frame pin was absent, whereas on the Liberator 2, the complete barrel was missing. Liberator 5 was particularly affected by the discharge: the barrel was missing, as well as two frame pins. A third frame pin was also displaced, and the frame cracked.

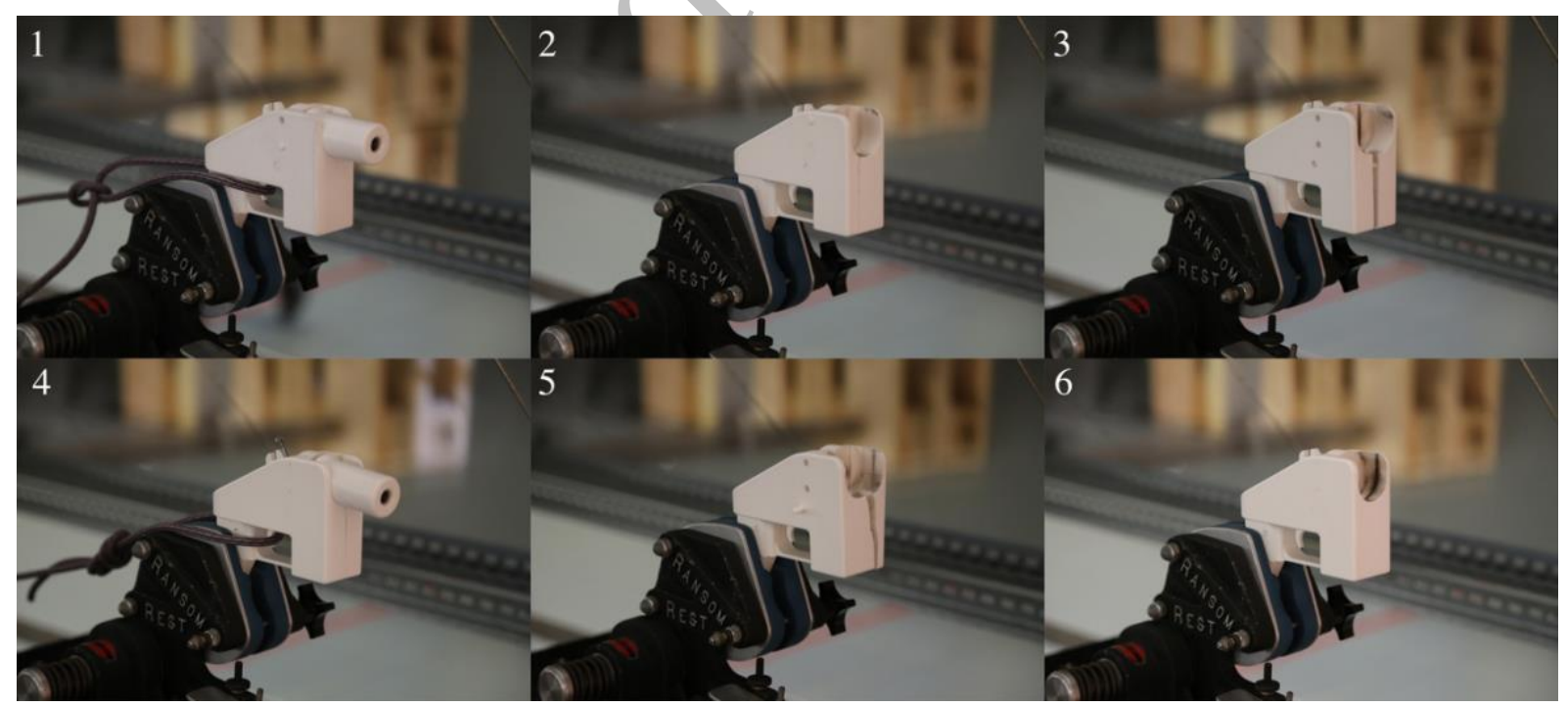

Figure 4: State of the Liberators after discharging one round.

Depending on the barrel's condition, the cartridge case was either found inside it - if the barrel remained intact - or ejected when the barrel broke. Polymer pieces, mostly originating from the barrel, were ejected away from the printed handgun, especially when the barrel broke during the shooting process. 
High-speed camera recordings revealed that the fracture of the barrel occurred when the bullet was still inside the firearm (Figure 5).

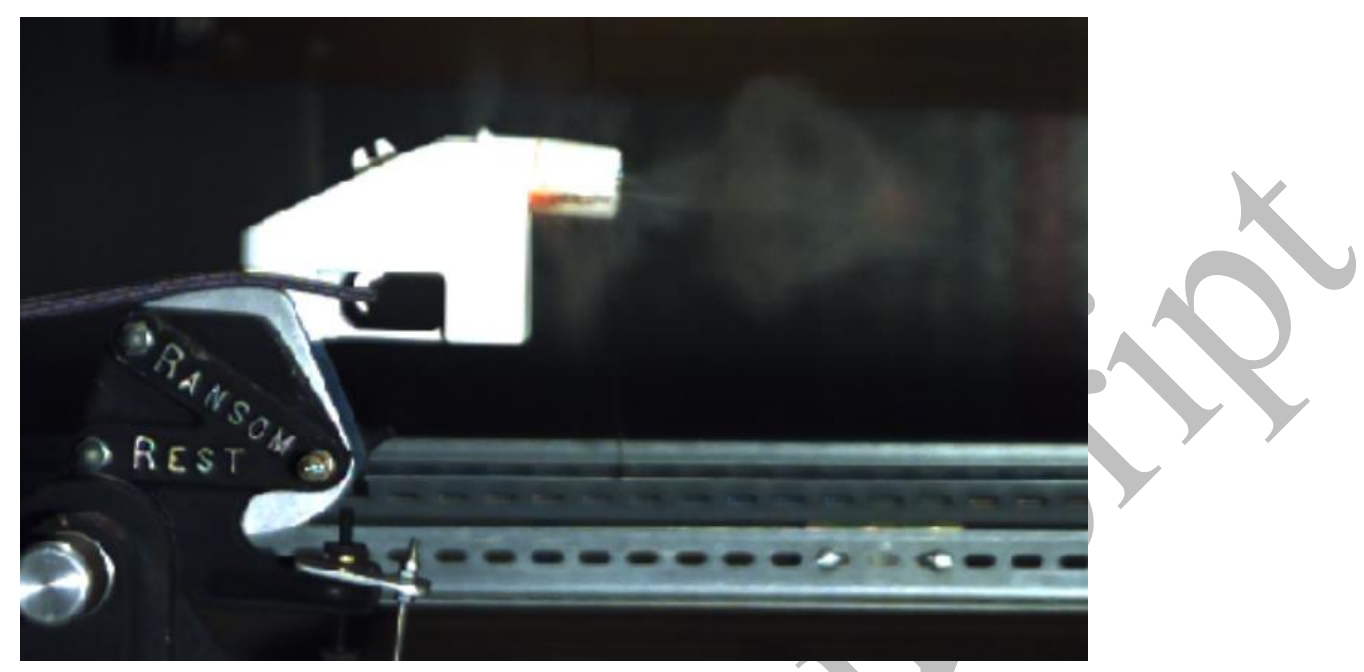

Figure 5: Snapshot from the high-speed camera recording of the discharge of the Liberator 2. The gases are scattering around the broken barrel, not only from the muzzle.

The calibres used in this study have lesser maximum pressure values than the most common calibres such as $9 \mathrm{~mm}$ Luger. However, the ABS barrel of the Liberator cannot support the pressure of the expanding gas. Compared to usual firearms, the layered polymer structure of the barrel weakens the strength of the piece, causing it to break most of the times.

\subsection{Speed and behavior of the bullets}

The two measures performed by shooting 380 Auto ammunition with a Beretta 86 were of 298 and $302 \mathrm{~m} / \mathrm{s}$. As indicated in Table 2, exit velocity values obtained with a Liberator for shots $\mathrm{N}^{\circ} 1$ to 4 are 40 to $60 \%$ lower than values obtained with a classical handgun for the same calibre. Besides, when the barrel remained intact, the exit velocity was about $30 \mathrm{~m} / \mathrm{s}$ higher compared to the measurements made when the barrel broke.

These results are in accordance with the hypothesis that the loss of energy due to the scattering of gases leads to a lower speed of the projectile. The difference of speed according to the condition of the barrel also supports this hypothesis. Indeed, if the barrel breaks, less energy is available to propel the projectile forward, resulting in a lower muzzle velocity. On the other hand, if the barrel remains intact, the pressure is concentrated on the projectile, resulting in a higher muzzle velocity.

In terms of trajectory analysis, the first discharge resulted in a projectile pointing forward. However, the five subsequent recordings revealed that all other projectiles were tumbling, bringing the tip of the projectile backwards. A video of a discharge is available in the Appendix.

For comparison purposes, Figures 6 and 7 illustrate the six trajectories of projectiles fired with Liberators and one of a projectile fired with the CZ 45. 


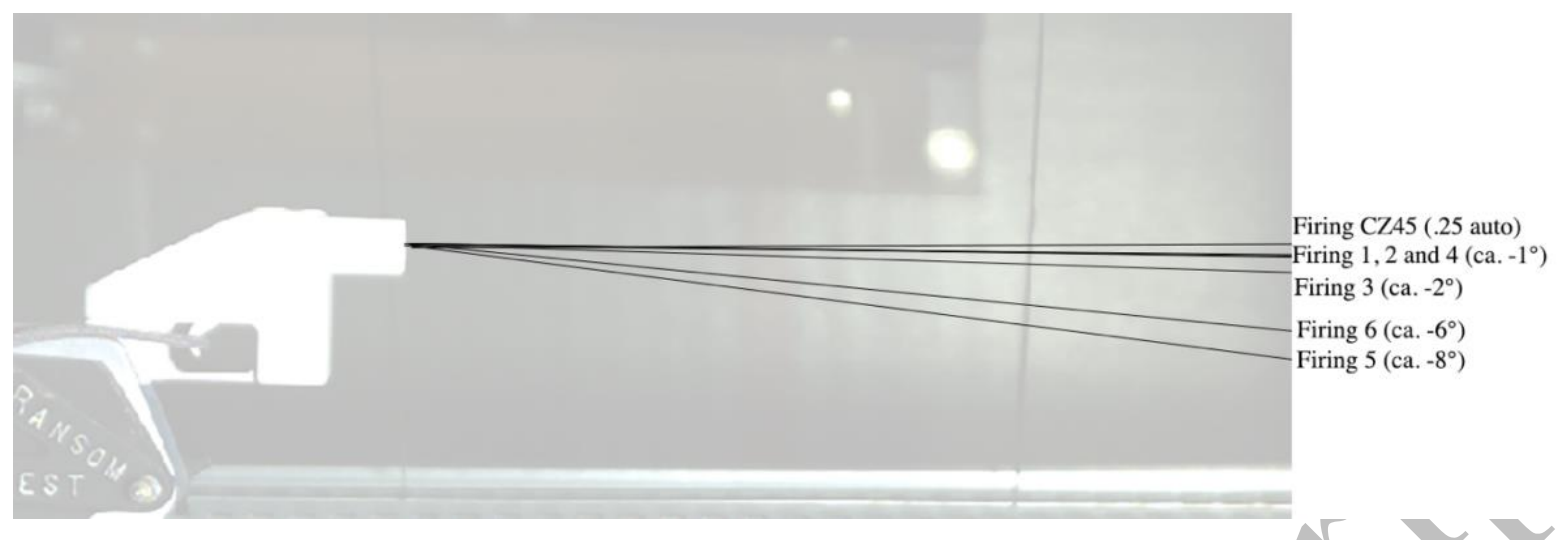

Figure 6: Vertical trajectories of the six projectiles fired with Liberators compared to the trajectory of a projectile fired with a .25 Auto calibre pistol.

Figure 6 highlights the disrupted trajectory ${ }^{5}$ for two out of the six discharges, which was more than $5^{\circ}$ lower than the projectile fired with the CZ 45 (number 5 and 6). The four other trajectories were closer to a classical trajectory. It is worth noting that the breaking of the barrel produced disrupted trajectories for both cases. Figure 7 also shows that projectile 5 had the greatest horizontal deviation. However, despite a broken barrel, projectile 6 had the smallest horizontal deviation out of the six. Projectiles 2 and 3 had vertical trajectories closer to the one of a classical firearm, even if the barrel broke in those cases. Their horizontal deviations also differed.

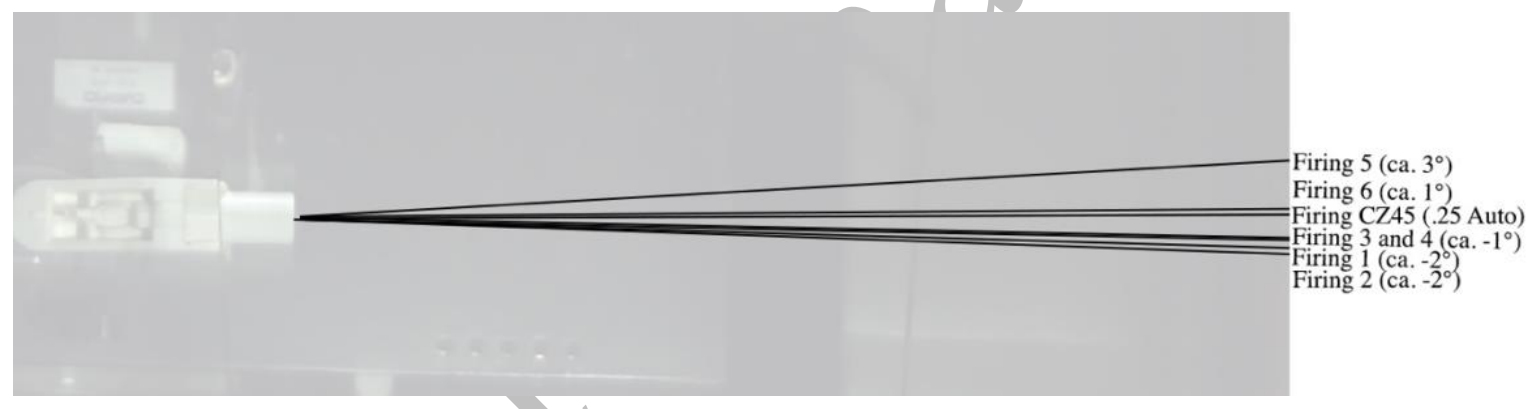

Figure 7: Lateral trajectories of the six projectiles fired with Liberators compared to the trajectory of a projectile fired with a .25 Auto calibre pistol, view from over.

These observations suggest that disruption of the trajectory is due to a combination of several factors. Among them are the change of direction of the gases when the barrels break (as gases have an influence on the projectile's intermediate ballistics [10]) and the rupture of the barrel, which could give a new direction to the projectile. The difference of velocity, compared to a classical firearm, may also explain the difference of trajectory.

Finally, the recordings of the six discharges show an unstable flight of variable magnitude. Since the barrel of the Liberator is not rifled, the projectile does not benefit from the usual gyroscopic stability, causing an unstable flight.

${ }^{5}$ Precautions were taken to ensure the measures were relevant, such as locking the rest and checking the alignment of the barrel while processing the images 


\subsection{Impact on the target}

Conventional GSR were observed on the adhesive sheet. Indeed, a significant number of unburnt particles were caught on the adhesive. However, some unusual perforations were also detected on the adhesive surface when a Liberator was discharged. Such perforations were not observed when the CZ 45 was discharged in the same conditions. Fragments of broken polymer pieces seem to be have gone through the adhesive target. This hypothesis is corroborated by the high-speed camera recordings (see Appendix for a video example). Besides, some of these fragments were found on the ground between the firearm and the target. This hypothesis is also in line with a previous study showing that polymer particles might be found among conventional GSR [7]. Figure 8 shows three adhesives sheets used to collect GSR: it includes (1) the discharge of the CZ 45 pistol, (2) the one of a .25 Auto Liberator and (3) the one of a .380 Auto Liberator. The black dots show GSR, the white dots show holes in the adhesive. Given that the CZ 45 and the Liberator have approximately the same barrel length, their GSR patterns are comparable [11].

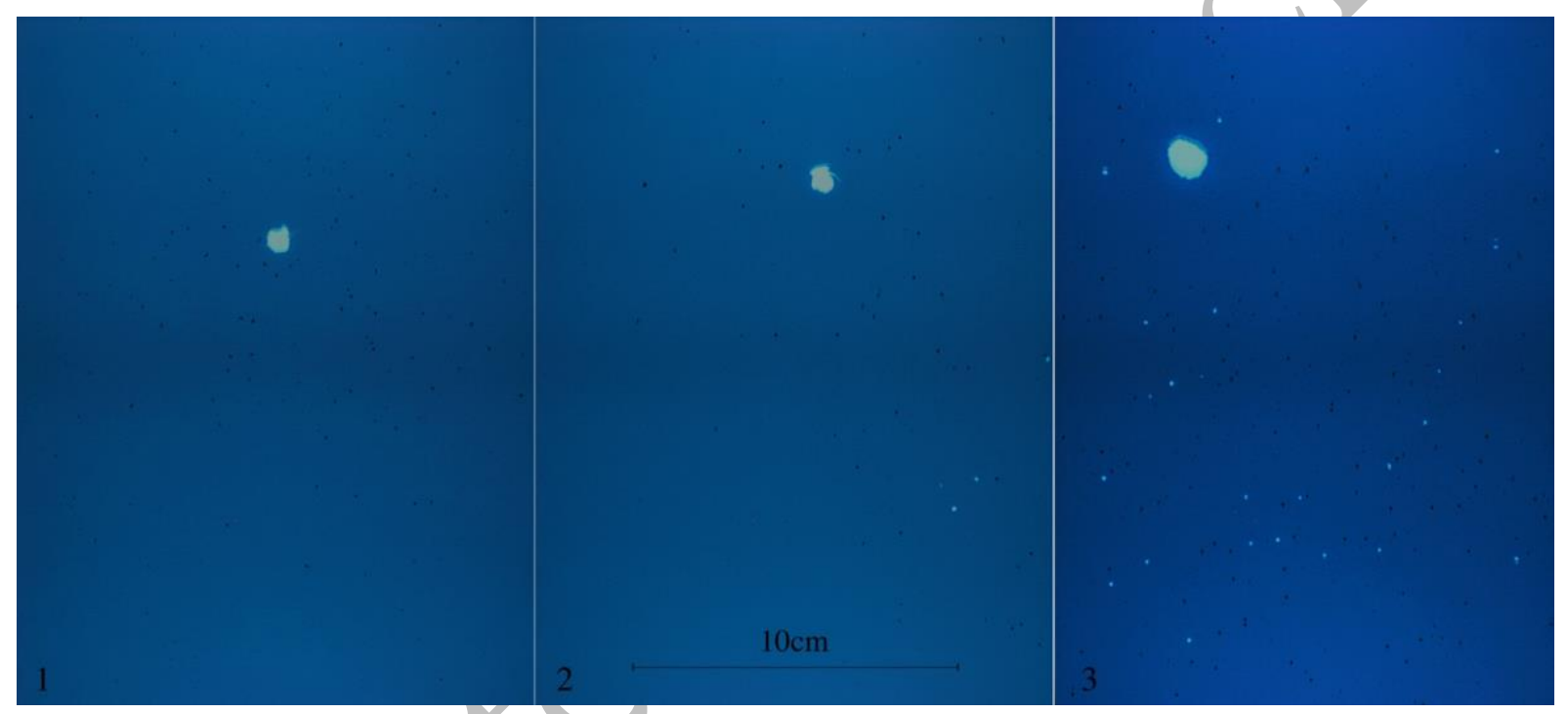

Figure 8: Adhesives sheets under a short wavelength UV light (254nm). 1: discharge of the CZ 45 (cal. .25 Auto). 2: discharge of the Liberator 6 (cal. .25 Auto). 3: discharge of the Liberator 5 (cal. .380 Auto). Note that the trajectory of the Liberator 5 was disrupted, which explains the entry hole on the side of the adhesive.

Figure 8 shows that for a given calibre, the discharge of the Liberator produced less GSR particles on the adhesive than the discharge with the CZ 45. In all cases, GSR particles tended to concentrate around the entry hole. A few perforations caused by polymer fragments were noticed with the .25 Auto Liberator. These perforations increased when using the .380 Auto Liberator. The .380 Auto being a more powerful ammunition could explain this observation. A more powerful ammunition causes more damage to the Liberator pieces, propelling more polymer debris and more GSR. Interestingly, on the adhesive sheet number 3 (.380 Auto Liberator) GSR seemed to be distributed on a wide area under the entry hole. This distribution should be compared with the GSR pattern of a .380 Auto calibre semi-automatic pistol having a similar barrel length (about $64 \mathrm{~mm}$ ). Such a firearm was not available for this study.

Wound ballistics is focusing on the effect, in terms of energy transfer and depth of penetration, caused by a projectile. As shown in Table 2, only two projectiles were found in the ballistics soap. Figure 9 shows the abovementioned effect when the barrel remained intact (discharge 4). Conversely, Figure 10 illustrates the cavity when the barrel broke (discharge 2). The depth of penetration of the recovered projectiles were respectively $21 \mathrm{~cm}$ and $14 \mathrm{~cm}$. 
Figures 9 and 10 reveal similarities with existing studies on wound ballistics. Both wounding patterns were comparable to those caused by heavy fragments [10]. Such channels were characterised by a deep penetration, most of the energy being transferred at the entrance of the wounding track.

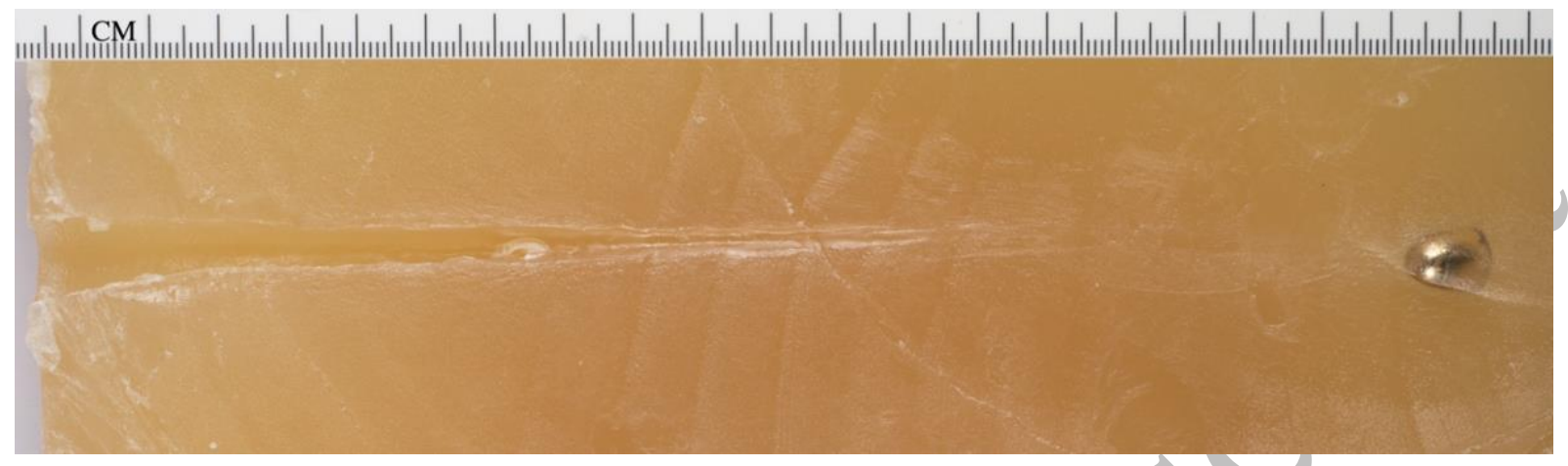

Figure 9: Cavity in the ballistics soap target, projectile 4.

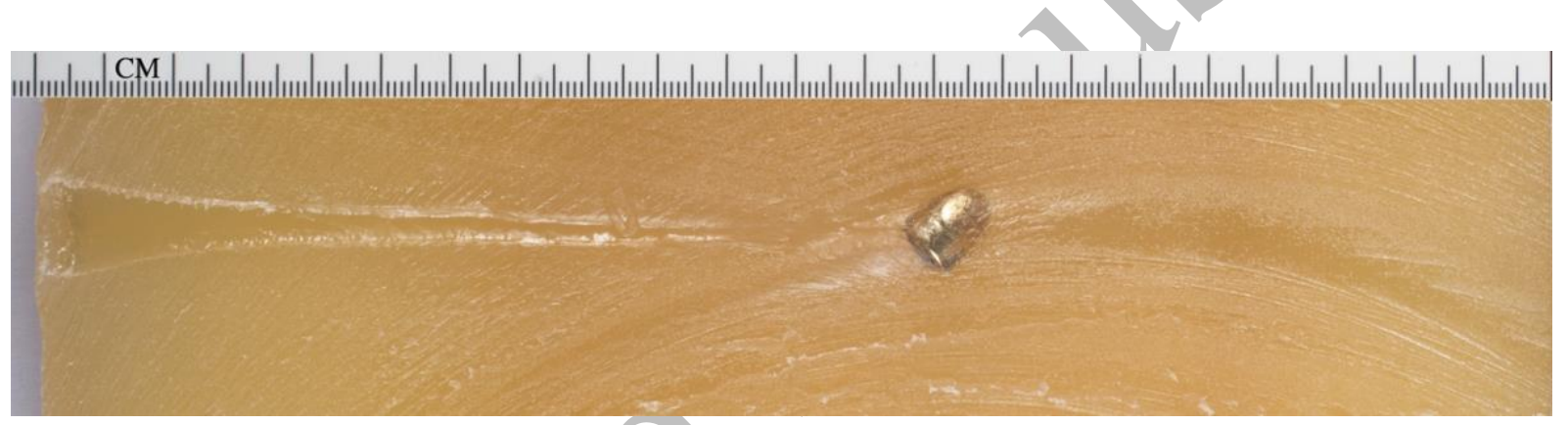

Figure 10: Cavity in the ballistics soap target, projectile 2.

As illustrated in Figure 9, the tip of the projectile is pointing backwards. Such an orientation of the projectile is not expected with a classical firearm. The high-speed camera recordings showed an absence of spin that might explain the behaviour of the projectile - knowing the projectile did not ricochet. To our opinion, the difference of muzzle velocity of the two projectiles - respectively 138 and $172 \mathrm{~m} / \mathrm{s}$ - explains the different depth of penetration shown in Figures 9 and 10.

\section{General discussion and conclusions}

This study aimed at developing a test-firing process, in order to analyse the discharge of polymer firearms. Only one model of 3D-printed handgun was considered, the Liberator. While providing very interesting information, this has to be emphasized as a limiting factor. Besides, a high-end 3D-printer was used to print the weapons, as well as a modified ABS adapted to the 3D-printer. These aspects tend to limit the generalisation of the results to other types of 3D-printed firearms, or to firearms printed with devices of lesser quality.

Firing six Liberators showed a wide range of observations, notably regarding the situation of the weapon after the discharge, the speed and behaviour of the projectiles and the impact on the target. Besides these variations, it is still possible to draw a few conclusions. First, the experiments proved that it is possible to discharge a Liberator, though important damages are sometimes caused to the weapon itself. Secondly, the projectile can reach its target despite its lack of precision, causing wounds in line with those of fragments. As a third point, polymer fragments 
and pieces of the weapons are ejected in the environment when discharging a 3D-printed Liberator. Projectiles and cartridge cases themselves might also display traces of polymer. When investigating a scene, if the use of a 3D-printed weapon is suspected, knowledge about this kind of traces is crucial for preservation and reconstruction purposes. In that sense, the second part of this series of articles will characterise these specific traces left near the weapon, on the Liberator itself and on the elements of ammunition.

\section{References}

[1] B. King and G. McDonald, "Behind the Curve, New Technologies, New Control Challenges." Small Arms Survey, 2015.

[2] G. Walther, "Printing Insecurity? The Security Implications of 3D-Printing of Weapons," Science and Engineering Ethics, vol. 21, no. 6, pp. 1435-1445, 2015.

[3] B. Lavergne and M. Lavergne, L’imprimante 3D, Une révolution en marche. Favre, 2014.

[4] Rhumorbarbe Damien, Werner Denis, Gilliéron Quentin, Staehli Ludovic, Broséus Julian, and Rossy Quentin, "Technical Note: Characterising the online weapons trafficking on cryptomarkets," Forensic Science International, vol. vol 283, pp. 16-20, 2018.

[5] A. Greenberg, “Meet The 'Liberator': Test-Firing The World's First Fully 3D-Printed Gun,” Forbes, $05-M a y-2013$. [Online]. Available: http://www.forbes.com/sites/andygreenberg/2013/05/05/meet-the-liberator-test-firing-the-worldsfirst-fully-3d-printed-gun/. [Accessed: 24-May-2017].

[6] R. J. Reilly, “Feds Printed Their Own 3D Gun And It Literally Blew Up In Their Faces," Huffington Post, 13-Nov2013.

[7] C. Crowe, "The Analysis of Gunshot Residue and Plastic Deposits From 3D Printed Polymer Firearms," presented at the American Academy of Forensic Sciences, New Orleans, United States of America, 2017.

[8] O. Black, R. Cody, D. Edwards, and J. V. Cizdziel, "Identification of polymers and organic gunshot residue in evidence from 3D-printed firearms using DART-mass spectrometry: A feasibility study," Forensic Chemistry, vol. 5, 2017.

[9] C. K. Chua, K. F. Leong, and C. S. Lim, "Rapid Prototyping Data Formats," in Rapid Prototyping: Principles and Applications, Singapore: World Scientific, 2010.

[10] B. Kneubuehl, R. Coupland, M. Rotschild, and M. Thali, Wound Ballistics Basics and Applications (translation of the 3rd German edition), 1st ed. Springer-Verlag Berlin Heidelberg, 2011.

[11] F. C. Barnes and R. A. Helson, "An Empirical Study of Gunpowder Residue Patterns,” Journal of Forensic Sciences, vol. 19, no. 3, 1974. 